\title{
DYNAMICS OF RUPTURE AT FRICTIONAL ROUGH INTERFACES DURING SLIDING INITIATION
}

\author{
Mariano Di Bartolomeo* \\ Department of Mechanics and Aeronautics \\ University of Rome 'La Sapienza', Italy \\ Mariano.DiBartolomeo@uniroma1.it
}

\author{
Francesco Massi \\ Laboratoire de Mécanique des Contacts et des \\ Structures, INSA-Lyon, Lyon, France. \\ francesco.massi@insa-lyon.fr
}

\author{
Anissa Meziane \\ Lab. de Mécanique Physique \\ Université de Bordeaux 1 \\ Talence, France \\ a.meziane@Imp.u-bordeaux1.fr
}

\author{
Laurent Baillet \\ Lab. Geophys. \& Tectonophys. \\ Joseph Fourier University, \\ Grenoble, France. \\ laurent.baillet@ujf-grenoble.fr
}

\author{
Antonio Culla \\ Dep. of Mech. and Aeronautics \\ University of Rome 'La \\ Sapienza', Italy. \\ antonio.culla@uniroma1.it
}

\section{ABSTRACT}

The aim of this work is to present the results from a non linear finite element analysis in large transformations of the contact interface between two deformable bodies when sliding initiates and the roughness is introduced at the contact surfaces.

The two-dimensional in-plane dynamic model consists of two different isotropic elastic media separated by an interface governed by Coulomb friction law, and subject to remotely applied normal and shear tractions (pre-stress phase). Once the ratio between the local values of tangential and normal stresses reaches the limit value, the sliding initiates and local ruptures are activated (nucleation phase). The propagation of the ruptures over the interface and the wave propagation inside the solids are analyzed. The interactions between the waves propagating into the two solids ( $P$ waves, shear waves, surface waves) give raise to different types of ruptures. They can be classified depending on their velocity front (sub-Rayleigh, subshear, super-shear) or on their interface states (pulse-like, crack-like).

A sinusoidal roughness is introduced at the contact surfaces and the analysis is performed for different values of the roughness parameters. Depending on the relative dimension between the roughness wavelength and the width of the wave fronts, two different behaviour can be observed: i) a coupling between the wave propagating into the two bodies; ii) a decoupling of the wave propagation inside the two materials, characterized by an independent wave propagation.
First the wave propagation is analyzed when a single rupture is originated in pre-sliding conditions; successively, the wave generation during sliding initiation is addressed.

\section{INTRODUCTION}

Wave generation and propagation from contact interfaces is a substantial subject of interest for researchers involved on several domains, mainly tribology, and dynamics. In the last few years several papers presented models considering wave generation from a dynamically extending shear rupture [1-3], where a rupture is considered when an initially sticking zone of the interface is becoming in sliding state. The different ruptures can be classified depending on the velocity of their front [3]: (i) Sub-shear type: the velocity of the rupture front is less than the shear wave velocity; (ii) Super-shear type : the velocity of the rupture front is more than the shear wave. They can also be classified depending on the interface states [3]: (j) Pulse-like rupture: only a small part of the broken interface is in sliding state; (jj) Crack-like rupture: the broken part of the interface is sliding continuously.

Different types of ruptures have been investigated: subRayleigh pulse-like, sub-Rayleigh crack-like, super-shear pulse-like and supershear crack-like ruptures. The ruptures analyzed in such works nucleate in a pre-stressed state corresponding to the sliding initiation between the two surfaces in contact.

\footnotetext{
* Address all correspondence to this author:

Department of Mechanics and Aeronautics, University of Rome 'La Sapienza'

via Eudossiana 18, 00184 Rome, Italy, fax. +39 06 484854, phone +390644585556
} 


\subsection{Dynamic Ruptures in Mode II}

There is a fundamental theoretical difference between ruptures in mode II (the slip is parallel to the direction of rupture propagation) along the interface that separates similar or dissimilar materials [4]. On a planar fault between identical media symmetry implies that there is no coupling between slip and normal traction, if the friction law is a classical Coulomb friction law. If there is a material contrast across the fault, the symmetry is broken and slip can modify the normal stress. Weertman [5] suggested that a dynamic reduction of the normal interface stress may allow a slip pulse to propagate at about the slower shear wave speed, even if the interface is governed by a Coulomb friction law. Such a dynamic reduction of the normal interface stress can be due to material contrast, heterogeneous stress state or asperities. Andrews et al [4] studied the selfsustaining propagation of a slip pulse associated with dynamic changes of normal stress, in the case of a contrast of $20 \%$ in elastic wave speed. Their numerical results confirmed the prediction of Weertman. If the Rayleigh Generalized (RG) wave speed exists, a slip pulse propagates at RG wave speed along the slip direction of the softer media. Otherwise, the slip pulse propagates in the same direction at the weakest shear wave velocity of the two media. For a sufficiently high friction coefficient a self sustained pulse propagating at the slower $\mathrm{P}$ (longitudinal) wave speed in the opposite direction, the slip direction of the stiffer media, can also occur [6].

Adams [1] generalized Weertman's results in super-shear regimes. He showed that a pulse-like rupture between two dissimilar media exists with a constant friction coefficient at the interface if the contrast between the two media is not too important. The pulse existence is presented as a function of different parameters like contrast, friction coefficient and nucleation length.

\subsection{Effect of Roughness}

The roughness of the contacting surfaces plays an important role in determining the nature of the shear stress distribution and consequently affects the rupture dynamics.

When two bodies are pressed together, because surfaces are rough, the real area of contact ("fractional contact area") is much smaller than the apparent contact area. Various models of contacting rough surfaces have been proposed in the literature; the work by Oden and Martin [7] gives an extensive overview of these models. The fractional contact area bears the entire applied macroscopic load, such that a heterogeneous distribution of the local normal stress is produced at the interface between the bodies; if the contact is subjected to a tangential force, also the local shear stress distribution is heterogeneous. The presence of asperities generates a complex rupture process and, because of stress concentrations, causes abrupt changes in slip velocity, which are the source of high frequency waves [8]. When the crack tip crosses the contact zone, it can interact with the asperity in three different ways [9]: i) the asperity is broken as the crack tip passes; ii) the crack tip proceeds beyond the barrier, leaving behind unbroken asperity; iii) the asperity is not broken at the initial passage of the crack tip but it has an increase in dynamic stress and eventually breaks with the passage of secondary rupture fronts. Rubinstein et al [10] investigated the dynamics of the incipient sliding between rough surfaces. They showed that the onset of motion is preceded by discrete sequence of crack-like precursors, which are initiated at shear level that are below the threshold of static friction. These precursors redistribute the true contact area along the interface and consequently the local stress. In the present work the analysis of the effect of the interface asperities is developed. The simulations show that, due the heterogeneous stress distribution, a differentiated rupture develops inside the asperity with different front velocities at different portions of the asperity; during the process, changes of the front rupture velocity may occur. The conditions for coupling or uncoupling between the waves radiating in the two bodies have also been investigated. Their correlation to the relative dimension between the roughness and the width of the wave fronts has been observed.

The aim of this work is to present a non linear finite element analysis in large transformations of: a) the dynamic rupture, in pre-sliding conditions, at the frictional interface between two deformable bodies with and without roughness. b) rupture and wave propagation during sliding initiation between two bodies. The paper is organized as follows: section 2 presents the mathematical formulation on which the explicit dynamic finite element code (PLAST2) used to perform the simulations is based; the numerical model and the relative assumptions are also described. In section 3 ruptures between two bodies in pre-sliding conditions are investigated. In section 4 wave propagations during sliding initiations is analyzed. The results are finally summarized in section 5 .

\section{NUMERICAL MODEL}

\subsection{Finite Element Model}

PLAST2, an explicit dynamic finite element code, is used to simulate the dynamic behaviour of two bodies $\left(P^{(1)}\right.$ and $P^{(2)}$ in Figure 1) during frictional contact. PLAST2 is designed for large deformations and non-linear contact behaviour. It uses a forward Lagrange multiplier [11] method for the contact between deformable bodies. For the dynamic simulation, the formulation is discretized spatially by using the finite element method and discretized temporally by using the $\beta_{2}$ method. The contact algorithm uses slave nodes (situated at $P^{(l)}$ contact surface) and target surfaces (at the $P^{(2)}$ contact surface) described by a four node quadrilateral element with $2 \times 2$ Gauss quadrature rule. The elementary target segments are described by two nodes and approximated by bicubic splines [12].The forward Lagrange multiplier method is formulated by equations of motion at time $\left(t^{i}=i \Delta t\right)$ with the displacement conditions imposed on the slave nodes at time $t^{i+1}$ : 


$$
\left\{\begin{array}{l}
\mathbf{M} \ddot{\mathbf{U}}^{i}+\mathbf{C} \dot{\mathbf{U}}^{i}+\mathbf{K} \mathbf{U}^{i}+\mathbf{G}^{i+1^{\mathrm{T}}} \boldsymbol{\lambda}^{i}=\mathbf{F}^{i} \\
\mathbf{G}^{i+1} \mathbf{U}^{i+1} \leq 0
\end{array}\right.
$$

Where $\mathbf{M}, \mathbf{C}$ and $\mathbf{K}$ are respectively symmetric and positively defined matrices of mass, Rayleigh's proportional damping $\left(\mathbf{C}=d_{1} \mathbf{M}+d_{2} \mathbf{K}\right)$ and stiffness of the system.

$\mathbf{U}, \dot{\mathbf{U}}, \ddot{\mathbf{U}}$ are respectively the vectors of nodal displacements, nodal velocities and nodal accelerations. $\mathrm{F}$ is the vector of nodal external forces.

$\boldsymbol{\lambda}=\left[\begin{array}{ll}\lambda_{N} & \lambda_{T}\end{array}\right]^{\mathbf{T}}$ contains respectively normal and tangential forces at contact points $\mathrm{C}_{\mathrm{m}}$.

$\mathbf{G}^{\mathbf{T}}=\left[\mathbf{G}_{N}^{\mathbf{T}} \mathbf{G}_{T}^{\mathbf{T}}\right]$ is the global matrix of the displacement conditions ensuring non-penetration and contact law of the bodies in contact.

The equations of motion (1) are discretized in time, by using an explicit Newmark scheme. The vectors $\ddot{\mathbf{U}}^{i}$ and $\dot{\mathbf{U}}^{i}$

are expressed at each time step using a time scheme of type $\beta_{2}\left(\beta_{2} \in[0.5 ; 1[)\right.$ :

$$
\left\{\begin{array}{l}
\ddot{\mathbf{U}}^{i}=\frac{2}{\Delta \mathbf{t}^{2}}\left(\mathbf{U}^{i+1}-\mathbf{U}^{i}-\Delta \mathbf{t} \dot{\mathbf{U}}^{i}\right) \\
\dot{\mathbf{U}}^{i}=\frac{1}{1+2 \beta_{2}}\left\{\dot{\mathbf{U}}^{i+1}+\Delta \mathbf{t}\left(1-\beta_{2}\right) \ddot{\mathbf{U}}^{i-1}+\frac{2 \beta_{2}}{\Delta \mathbf{t}}\left(\mathbf{U}^{i+1}-\mathbf{U}^{i}\right)\right\}
\end{array}\right.
$$

The displacements ${ }^{*} \mathbf{U}^{i+1}$ of the nodes situated on the contact surface $\left(P^{(I)}\right.$ and $\left.P^{(2)}\right)$ are first computed with $\lambda^{\mathrm{i}}$ equal to $\mathbf{0}$.

A constraint matrix $\mathbf{G}^{\mathrm{i}+1}$ is formulated for the slave nodes if they have penetrated through a target segment. Calculations of contact forces $\lambda^{\mathrm{i}}$ and nodal displacement at time $t^{i+1}$ are then performed:

$$
\left\{\begin{array}{l}
\lambda^{i}=\left\{\Delta t^{2} \mathbf{G}^{i+1} \mathbf{M}^{-1} \mathbf{G}^{i+1^{\mathrm{T}}}\right\}^{-1} \mathbf{G}^{i+1}\left({ }^{*} \mathbf{U}^{i+1}\right) \\
\mathbf{U}^{i+1}={ }^{*} \mathbf{U}^{i+1}-\left(\Delta t^{2} \mathbf{M}^{-1} \mathbf{G}^{i+1^{\mathrm{T}}} \lambda^{i}\right)
\end{array}\right.
$$

Equations (3) are solved using the Gauss-Seidel method.

\subsection{Numerical Simulations}

The two-dimensional in-plane dynamic rupture model consists of two different isotropic elastic media separated by an interface governed by classic Coulomb friction law, i.e. no distinction between static and kinetic friction is made; the friction coefficient is equal to 1 .

Table 1 shows the material parameters used for the simulations; they have been selected according to the literature to assure the existence of the generalized Rayleigh wave [13]. Weertmann [5] showed that when such wave exists, a selfhealing slip pulse can propagate along the frictional interface at
Table 1. INPUT DATA, PROPAGATION VELOCITIES OF BULK WAVES AND RAYLEIGH WAVES IN $P^{(1)}$ AND $P^{(2)}$.

\begin{tabular}{lcc}
\hline & $P^{(l)}$ & $P^{(2)}$ \\
\hline Length & $20 \mathrm{~mm}$ & $20 \mathrm{~mm}$ \\
Width & $5 \mathrm{~mm}$ & $5 \mathrm{~mm}$ \\
Young modulus (E) & $5.3 \mathrm{GPa}$ & $3.392 \mathrm{GPa}$ \\
Density ( $\rho$ ) & $1246 \mathrm{Kg} / \mathrm{m}^{3}$ & $1246 \mathrm{Kg} / \mathrm{m}^{3}$ \\
Poisson ratio $(v)$ & 0.25 & 0.25 \\
Damping coefficient $d_{l}$ & $10 \mathrm{~s}^{-1}$ & $10 \mathrm{~s}^{-1}$ \\
Damping coefficient $d_{2}$ & 0 & 0 \\
P (longitudinal) waves $\left(c_{P}\right)$ & $2430 \mathrm{~m} / \mathrm{s}$ & $1940 \mathrm{~m} / \mathrm{s}$ \\
S (shear) waves $\left(c_{S}\right)$ & $1270 \mathrm{~m} / \mathrm{s}$ & $1120 \mathrm{~m} / \mathrm{s}$ \\
Rayleigh waves $\left(c_{R}\right)$ & $1190 \mathrm{~m} / \mathrm{s}$ & $950 \mathrm{~m} / \mathrm{s}$ \\
\hline
\end{tabular}

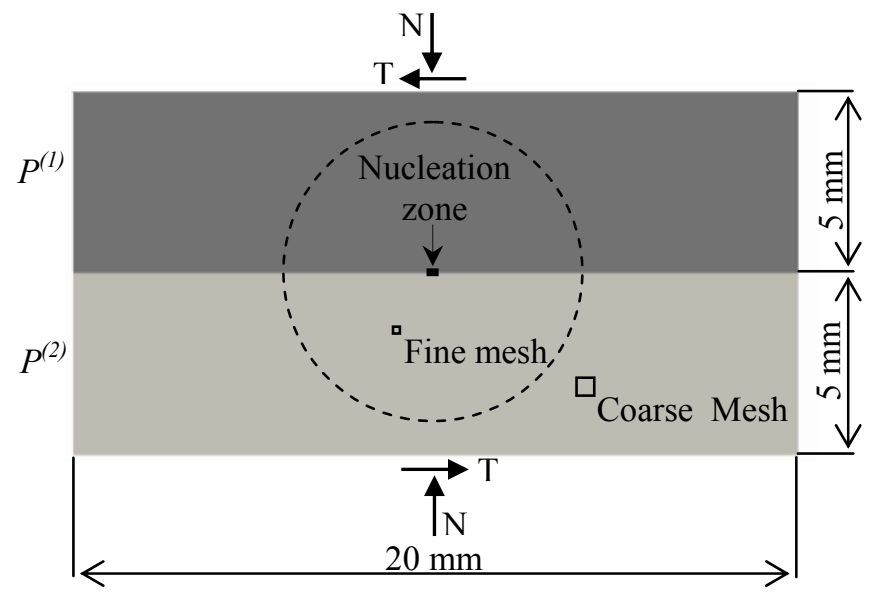

Figure 1. GEOMETRY OF THE 2-D BI-MATERIAL MODEL. TWO BODIES OF $5 \times 20 \mathrm{~mm}$. THE MESH CONSISTS OF A CENTRAL CIRCULAR REGION WITH FINE DISCRETIZATION (QUADRILATERAL ELEMENTS $0.0125 \times 0.0125 \mathrm{~mm}$ ) AND AN EXTERNAL REGION WITH WIDER MESH (QUADRILATERAL ELEMENTS $0.1 \times 0.1 \mathrm{~mm}$ )

generalized Rayleigh velocity in the slip direction of the compliant solid [2].

In the first part of the work (rupture in pre-sliding conditions) the two bodies are pressed together by a global normal force $\mathrm{N}$ applied on the external surfaces, and then subjected to a shearing force $\mathrm{T}$ which is calculated to be just less than the required one to produce slipping (Fig.1). The rupture is initiated in the "nucleation" zone at the centre of the contact interface, by decreasing instantaneously the friction coefficient to zero on the length $L_{n u c}$ (length of nucleation zone) corresponding to a defined number of contact nodes (Fig. 2); in such a way a rupture in mode II (the slip is parallel to the direction of rupture propagation) can be initiated. 




Figure 2. NORMAL (-) AND TANGENTIAL (----) STRESS AT THE NUCLEATION TIME ( $t=0 \mathrm{~s})$. THE TANGENTIAL STRESS BECOMES ZERO AT THE CENTRE OF THE INTERFACE (NUCLEATION NODES).

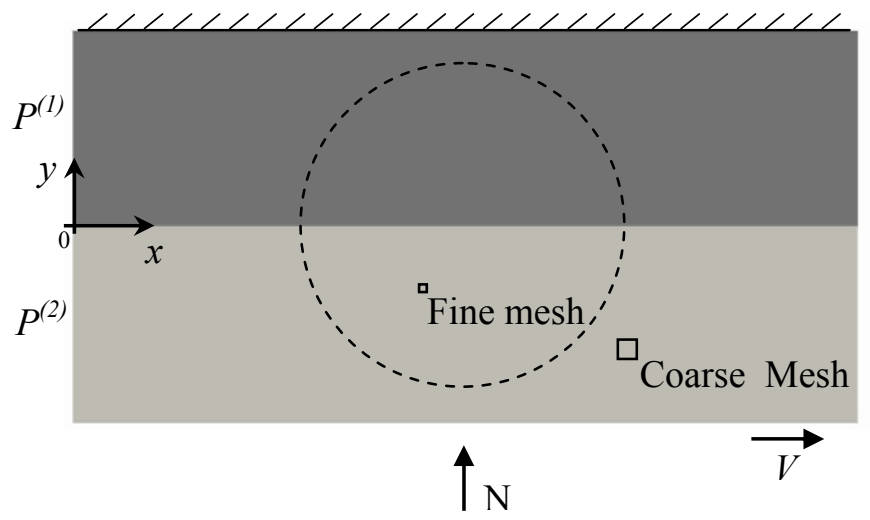

Figure 3. GEOMETRY AND BOUNDARY CONDITIONS OF THE 2-D BI-MATERIAL MODEL, IN THE CASE OF STUDY DURING SLIDING.

In the second part of the work (analysis of wave propagation during sliding initiation) the model is the same and the two bodies are pressed together by the same global normal force $\mathrm{N}$, but at the lower surface of $P^{(2)}$ is applied a constant velocity $V$, while the upper surface of $P^{(l)}$ is blocked (Figure 3). In such a way the sliding initiates and local ruptures are caused by the increase of the local tangential stress state, respect to the normal one.

\section{RUPTURE BETWEEN TWO BODIES IN PRE- SLIDING CONDITIONS}

\subsection{Rupture between Smooth Contact Surfaces}

This part presents the results stemming from the simulations of rupture along smooth interface separating two dissimilar materials. The dissymmetry induced by the difference in material properties causes a coupling between normal traction and tangential slip. Weertman [5] suggested that the dynamic reduction in compressive normal traction (due to the coupling phenomenon) may allow a slip pulse to propagate in a self-sustained manner at about the slower $\mathrm{S}$ (shear) wave speed. This part aims to examine the characteristics of pulse-like rupture of a dissimilar material interface governed with constant friction coefficient to validate the numerical model by comparison with the literature.

Figure 4 represents the isovalues of the velocity of the two bodies in contact. The nucleation zone centre is situated at $x$ equal to $10 \mathrm{~mm}$ and measures $0.125 \mathrm{~mm}$.

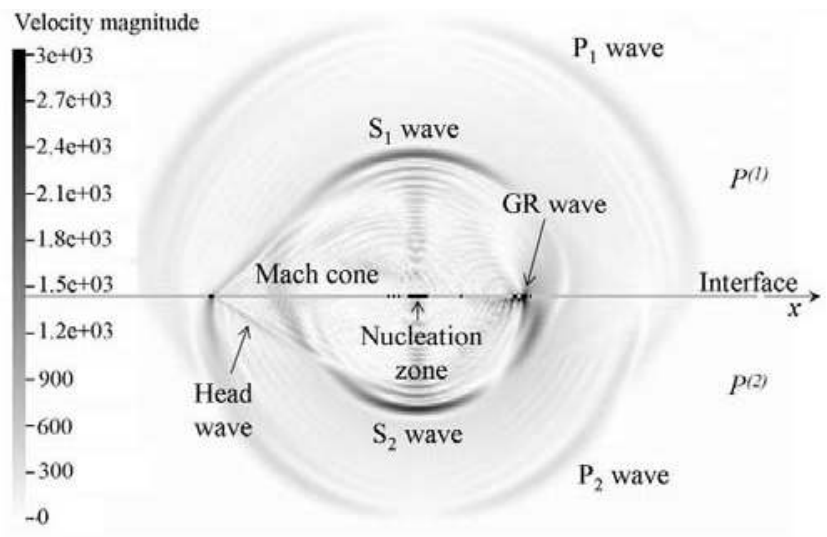

Figure 4. REPRESENTATION OF VELOCITY ISOVALUES $[\mathrm{mm} / \mathrm{s}]$. THE NUCLEATION ZONE CENTRE IS SITUATED AT $x$ EQUAL TO $10 \mathrm{~mm}$ AND MEASURES $L_{N U C}=0.125 \mathrm{~mm}$. SNAPSHOT SIZE $4.5 \times 3 \mathrm{~mm}$ THE POINTS OF THE INTERFACE IN BLACK ARE IN SLIDING.

The waves generated by the nucleation are identified: Pwaves and $\mathrm{S}$-waves in $P^{(l)}$ and $P^{(2)}$, and the generalized Rayleigh (GR) wave at the interface. As the wave velocities are different in $P^{(l)}$ and $P^{(2)}$, the nucleation induced different displacement on opposite side of the interface, causing changes in normal stress. Ben Zion [14] showed that these changes in normal stress are associated with a head wave propagating along the interface. Two rupture fronts propagating (left and right fronts) from the nucleation can be observed. At the left of the nucleation zone (Fig. 5(a)), the rupture front evanishes at the $\mathrm{x}$ position of $8.7 \mathrm{~mm}$. It corresponds to a decaying supershear pulse-like rupture $\left(c_{R U P} \approx 1700 \mathrm{~m} / \mathrm{s}\right)$. From cumulative sliding profile in figure 5(b), it can be seen that 

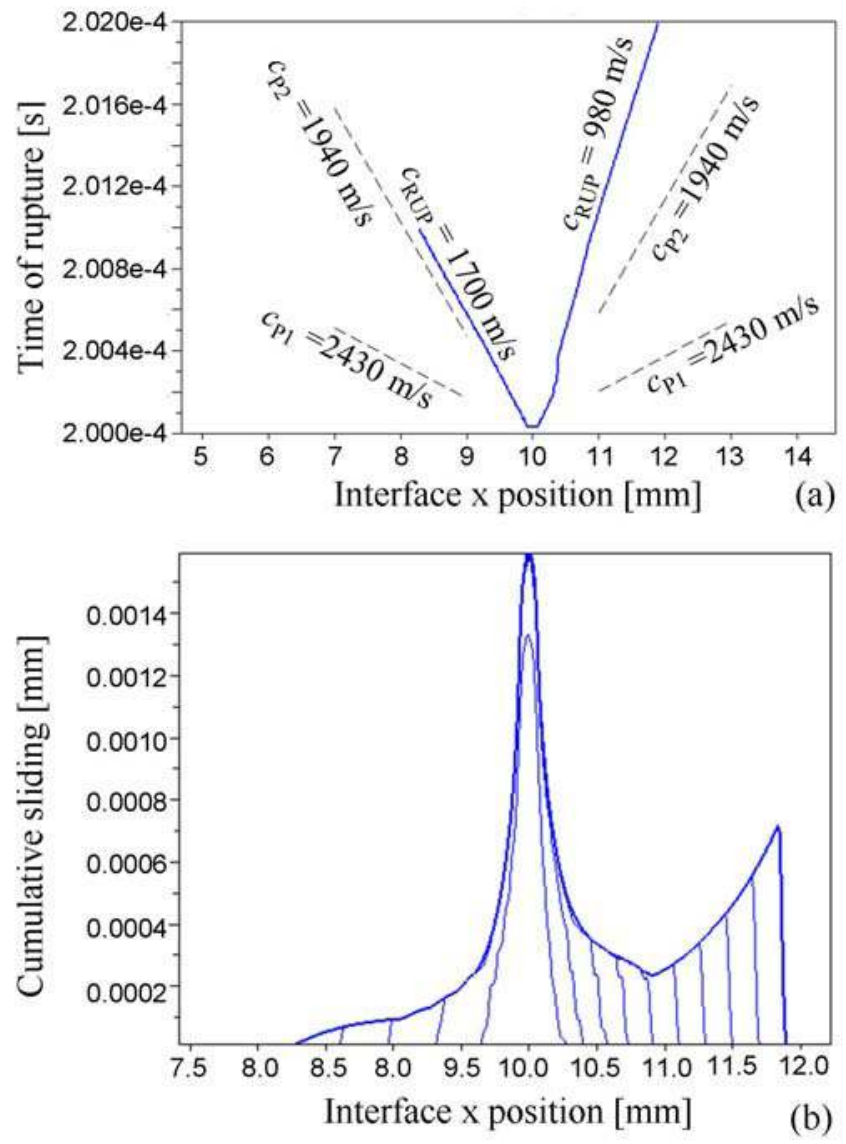

Figure 5 (a) RUPTURE PROFILE AND (b) CUMULATIVE SLIDING AS A FUNCTION OF $x$ POSITION ON THE INTERFACE, LnUc $=0.125 \mathrm{~mm}$ NUCLEATION ISTANT 2e-4 s. (REPRESENTATION OF CUMULATIVE SLIDING AT THE INTERFACE EACH 2e-7 s, FROM 2e-4 s TO 2.02e-4 s).

after the sliding induced by the front arrival, the sliding stops and after a short sliding time the contact zone is self-healing (sticking again). Along propagation, the sliding distance (figure $5(\mathrm{~b})$ ) and the relative sliding velocity at the interface are both decreasing (figure 5(b)) causing the decaying of the rupture.

The right-hand pulse, with respect to the nucleation zone, is a self-sustained sub-Rayleigh. The velocity of the rupture front is $c_{R U P} \approx 980 \mathrm{~m} / \mathrm{s}$ (near to $c_{R 2}=950 \mathrm{~m} / \mathrm{s}$ ). From figure 5 (b) it can be seen that, as for the left one, the rupture is self healing. Because the sliding distance is increasing along the propagation, this corresponds to the propagation of a sliding pulse in self sustained manner. These results are consistent with those of Ben Zion [4]. The nucleation causes the propagation of a slip pulse at about the Rayleigh wave velocity in the direction of imposed displacement of the slower velocity medium (in the positive $\mathrm{x}$ direction).

When the nucleation length increases the strain energy released increases. For example, for a nucleation length of $0.5 \mathrm{~mm}$, the left rupture is a self-sustained with velocity that tends to the velocity of $\mathrm{P}$ waves in the softer material $P^{(2)}\left(c_{P 2}\right)$, and the right one becomes a supershear crack [18]. The phenomenon of self-healing disappeared and as the rupture front passes by the part of broken interface continues to slide, causing more important friction dissipation than for a pulse-like rupture [1]. The obtained results agree with recent literature and allow for validating the numerical model used in this work.

\subsection{Rupture at Contact Surfaces with Asperities}

\subsubsection{Introduction of the Roughness}

The two profiles in contact have been modified assigning them undulations in order to introduce the asperities of a surface. The two bodies are pressed together by the same global force applied in the previous simulations. Sinusoidal profiles have been considered; it allows for keeping the focus on the relationship between the propagating waves in the bodies and the distribution and dimension of the asperities over the surfaces, function of the sinusoid length. Matching sinusoids with different spatial phases and amplitudes, four paths have been selected to be introduced at the interface. The 1st case, smooth surfaces, has already been discussed in section 3.1 In the 2nd case the sinusoidal profiles over the two contact surfaces are in phase, in the 3rd case they have a spatial phase difference of $\pi$ radians and in the 4th case there is roughness only over one of the two surfaces. Every roughness profile is characterized by the amplitude A and the spatial wavelength $\lambda$. For every pattern simulations with different values of $A$ and $\lambda$ and with different length of nucleation have been carried out .

\subsubsection{Coupled/Uncoupled Wave Propagation}

Figure 6 represents the isovelocities $(\mathrm{mm} / \mathrm{s})$ for two different values of the roughness wavelength with the pattern 3 and length of nucleation of $0.0125 \mathrm{~mm}$, at $6.8 \mathrm{e}-7 \mathrm{~s}$ after the nucleation. The contact points highlighted in black are in sliding while the grey ones are in adherence.

In the figure 6(a) it can be seen that the P-waves, S-waves, the head waves (P-S conversion) [15] and the Rayleigh waves radiate away from the rupture, in an independent (uncoupled) way along the two materials; they propagate symmetrically with respect to the vertical axis, meaning that there is no coupling between the waves propagating through the two bodies in contact. The rupture at the contact interface occurs (right and left to the nucleation zone) when the first wave reaches the asperities in contact so that there is a supershear rupture front (Fig. 7(a) and 7(b)) with velocity at about the longitudinal velocity of the stiffer material (Table 2). On the contrary in figure $6(\mathrm{~b})$, for a smaller length of the sinusoidal roughness, the waves propagating through the two bodies are coupled and the rupture profile on the left is different from the rupture profile on the right, similarly to the case without roughness; as for the flat contact surfaces the coupling is due to generation of dynamic changes of normal stresses that depends on the slip gradient, material properties and direction of rupture propagation [16]. 

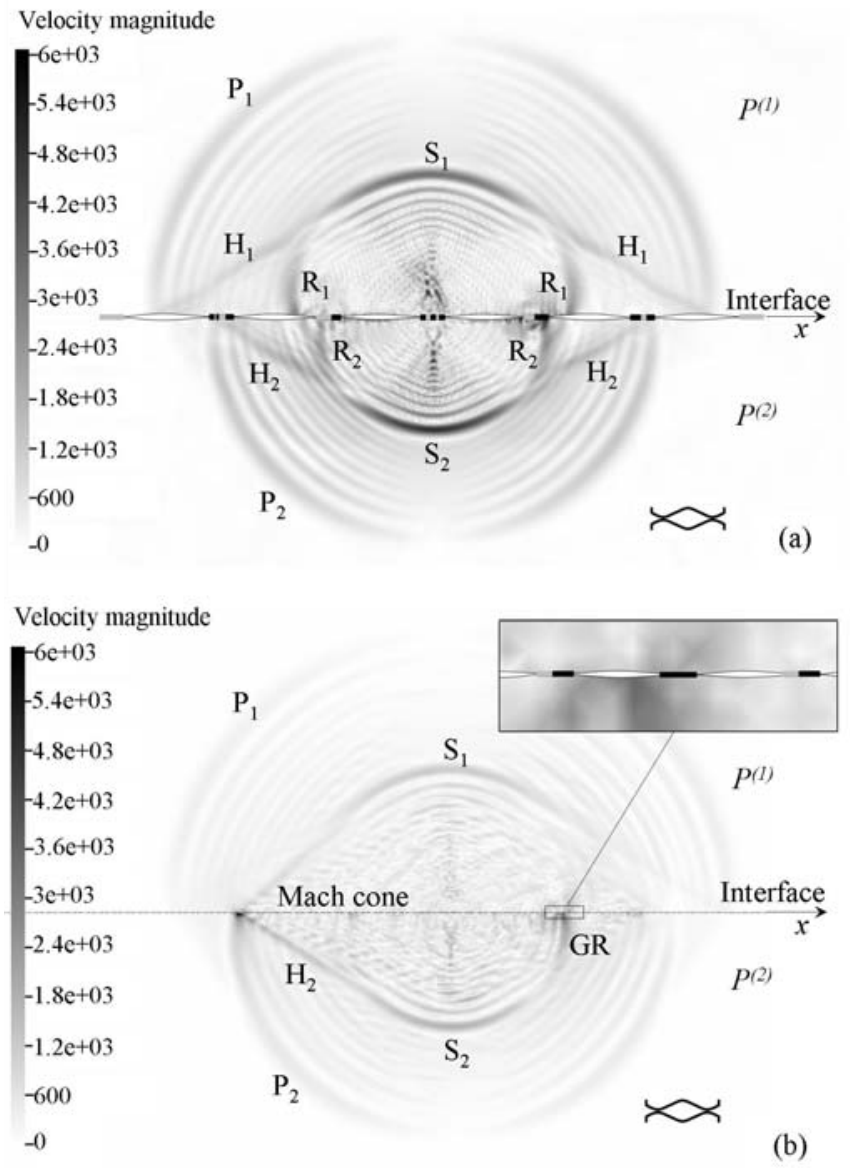

Figure 6. VELOCITY ISOVALUES [mm/s] AT 6.8e-7 s AFTER THE NUCLEATION, LENGTH OF NUCLEATION $0.0125 \mathrm{~mm}$, ROUGHNESS WITH $\pi$ SPATIAL PHASE DIFFERENCE. (a) $\lambda=0.6 \mathrm{~mm}, A=0.01 \mathrm{~mm}$. (b) $\lambda=0.075 \mathrm{~mm}, A=0.00125 \mathrm{~mm}$, IN THE INSET A MAGNIFICATION OF 10X. SNAPSHOT SIZE 4.5 x $3 \mathrm{~mm}$ THE POINTS OF THE INTERFACE IN BLACK ARE IN SLIDING.

To understand the uncoupling/coupling behaviour between the waves on the two bodies in contact, it is important to account for the width of the wave front, with respect to the spatial wavelength of the roughness. When they are of the same order of magnitude the waves can interact each other.

\subsubsection{Stress Distribution and Rupture Profiles.}

Because of roughness, it is not obvious to define the type of the rupture exactly. In a more general approach, it is known that the crack mode is favoured by relative high and smooth initial background stress and low or no coupling of slip to changes of normal stress (the mechanism described in section 3.1 ); while the pulse mode is favoured by opposite conditions [3].

As the rupture front moves, it continuously generates waves which are proportional to the local stress intensity factor and the instantaneous velocity of the rupture front [3].
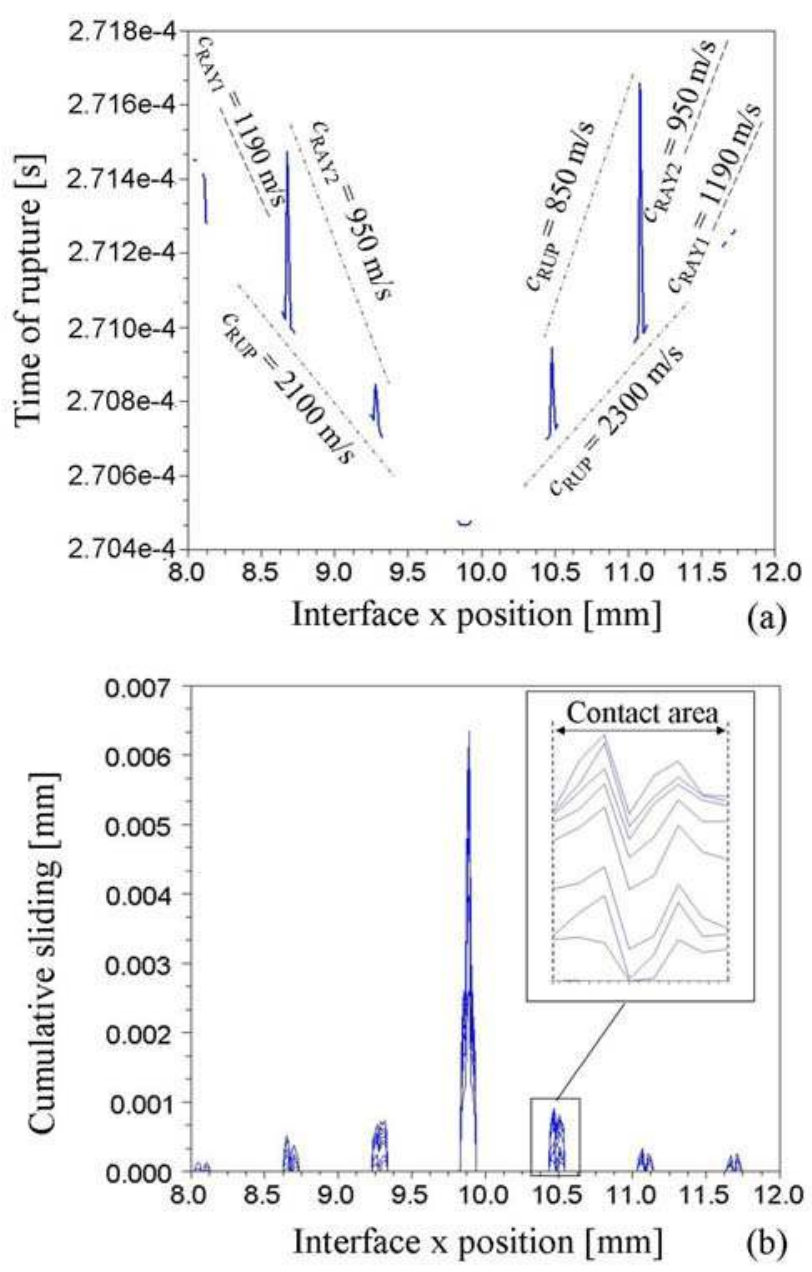

Figure 7. ROUGHNESS WITH $\pi$ PHASE DIFFERENCE, $\lambda=0.6$ $\mathrm{mm}, \mathrm{A}=0.01 \mathrm{~mm}$ (ref. Fig.6(a)). (a) RUPTURE PROFILE WITH $L_{\text {nuc }}=0.0125 \mathrm{~mm}$. (b) CUMULATIVE SLIDING PROFILE.

The presence of roughness, i.e. zones where the asperity are in contact and zones where there is no contact, brings to a heterogeneous shear and normal stress distribution, even inside the contact area, and causes a more complex rupture process.

Figure 7 shows the rupture profile relative to the case of figure 6(a); Keeping the attention, for example, on the first zone of contact at the right of the nucleation zone, when the first front of rupture (the longitudinal wave of the stiffer body) reaches the summit in contact, only the external points slide, while the central points, which have a lower local ratio between tangential and normal stress, do not slide Fig.6(a). Thus, the first rupture front skips the central part of asperity [9], because the energy is not sufficient for causing the sliding of the nodes where the tangential stress is lower. However, the first front causes a redistribution [10] of stresses along the asperity length. Such stress redistribution predisposes the points to slide more easily. The second wave that arrives on the asperity is the longitudinal wave of the softer body; the energy of this wave is still not sufficient to cause a global sliding on the asperities 
(Fig. 7(b) inset), even if a further change of the stress distribution is caused. Finally the nodes at this zone slip when the third wave, the Rayleigh wave of the upper body, arrives. This behaviour explains the feature of the rupture profile in figure 7(a), where two different velocity of propagation can be identified by connecting the external and internal points of the asperities. For examples on the right, for the external points of every asperity there is a rupture front with propagation velocity of $2300 \mathrm{~m} / \mathrm{s}$, while for the internal points $c_{R U P}=850 \mathrm{~m} / \mathrm{s}$. When the nucleation length is increased, the released energy is increased too; then, for causing the slipping of all the nodes of the asperity, the first and the second wave fronts are sufficient [18].

For the case in figure $6(\mathrm{~b})$, while the rupture of the whole two or three asperities close to the nucleation zone takes place with the arrival of the first wave, the rupture of the asperities away from the nucleation zone is differentiated.

\section{WAVE PROPAGATION DURING SLIDING INITIATION}

This part presents the analysis of the sliding initiation between dissimilar materials separated by a smooth interface. Contrarily to the previous section, the two surfaces start here to slide and the local ruptures (local sliding zones) are caused by the local ratio between tangential and normal stresses which passes over the friction coefficient value. The model is described in the last part of section 2.2 and the global tangential velocity applied to the lower body is $V=93.5 \mathrm{~mm} / \mathrm{s}$. The analysis is focused on very short time scales because, as it has been shown in the previous sections, the fracture occurs at speeds approaching the $\mathrm{P}$ waves of the bodies. Figure 8 represents the evolution of the contact force, more precisely the sum of the normal and tangential contact forces along the interface, during sliding initiation.

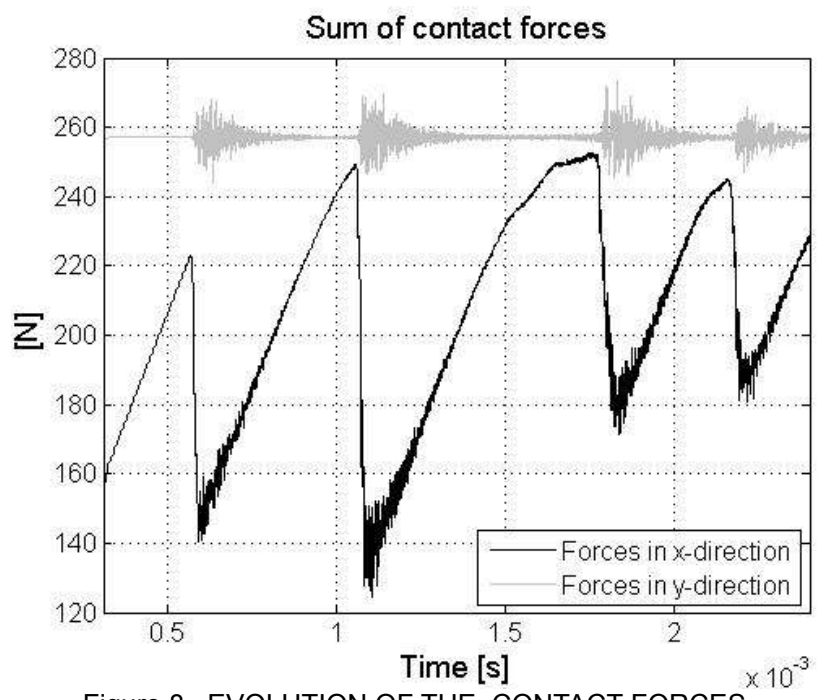

Figure 8. EVOLUTION OF THE CONTACT FORCES.
The first ramp of the tangential force (x-direction) grows until it reaches the value required to produce the first macrosliding which causes an instantaneous shoot of the global tangential force; the reason because it doesn't reach the value of the normal force (y-direction), despite the friction coefficient is 1 , is the non homogeneous normal stress distribution along the interface (Fig. 2). After the first ramp, the force drops down because of a macroscopic sliding and a following sequence of ramps and drops of the tangential force is generated (Fig. 8 and $10)$.

A different behaviour is observed between the first ramp and the following ones: i) In the former the interface comes from nearly steady-state conditions where all the contact nodes are in sticking state; consequently the global tangential force grows uniformly keeping the same slope until the drop occurs. ii) In the following ramps large oscillations of the global force are observed; such oscillations are due to the presence of waves generated at the local sliding zones (micro-slips) nucleated during the previous force drop (macroscopic sliding). These micro-slips play a key role to trigger the macro-sliding, behaving like "precursors" [10].

Defining "sliding" as a motion along the entire surface and "slip" or "micro-slip" as differential motion along part of the interface in according to [10], in the first macro-sliding (first force drop in Figure 8) the micro-slip occurs in detectable points of the interface, as shown in figure 9; the analysis shows that the waves are radiating from the local sliding zones, and every single zone behaves like a nucleated rupture (ref. Fig. 4); thus multiple nucleations occur simultaneously and the respective waves interact each other.

During the following ramps, an initial distribution of micro-slips [17] due to the previous macro-sliding, brings to different behaviour of the force ramp and the following drop (Fig. 8). The more important is the force drop (macro-sliding), longer is the time length of the following force ramp.

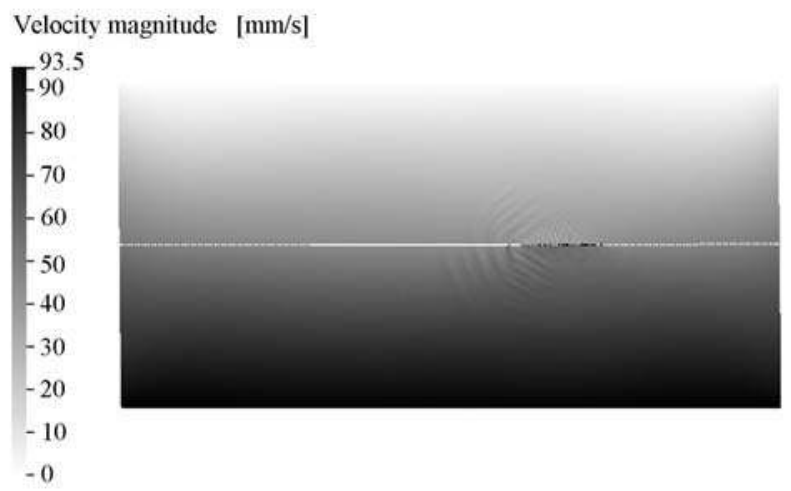

Figure 9. ONSET OF THE SLIDING: ISOVELOCITIES AT 0.5e3 s. THE POINTS OF THE INTERFACE IN BLACK ARE IN SLIDING. 




Figure 10. EVOLUTION OF THE FORCE OVER A LONGER TIME, 4.28e-3 s.

This is due to the fact that an important macro-sliding (large force drop) causes a large release of elastic energy, which has to be recovered before the next macro-sliding. As well, a larger force drop implies larger amplitude of the force oscillations during the following ramp.

Analyzing the evolution over a longer time, it can be seen (Fig. 10) that the amplitude of the force drops (and ramps) reduces and proportionally reduces the time length of the single ramp; moreover, the mean value of the tangential force increases and tends asymptotically to the normal force value. Focusing the attention on the single force ramp, variations of the ramp slope can be observed. Figure 11 shows a magnification of the previous diagram in figure 8 , around the interval between $1.1 \mathrm{e}-3 \mathrm{~s}$ and $1.9 \mathrm{e}-3 \mathrm{~s}$. Five characteristic zones can be identified: zone I, II,III and IV are characterized by an almost constant slope of the force, while the transition from one zone to the following is marked by an abrupt variation of the slope; zone V contains the force drop due to the macro-sliding.

Zone I): This zone is characterized by the presence of micro-slips, In the first part of this zone the percentage of interface in sliding (fig. 12, where status means the conditions at the interface) is about $4 \%$ and the $94 \%$ is in adherence, while a $2 \%$ is detached (a part of the detached surface is due to surface that discovers at the tips of the interface);

Zone II): at about $1.44 \mathrm{e}-3 \mathrm{~s}$, there is a first alteration of the slope, and this is confirmed by the figure 12 , where around $1.44 \mathrm{e}-3 \mathrm{~s}$ the percentage of the surface in sliding increases sharply.

Zone III): At about $1.5 \mathrm{e}-3 \mathrm{~s}$, there is more important knee in the force slope (Figure 12) and the percentage of the points in sliding has a remarkable growth approaching the $20 \%$

Zone IV): After a further fold of the force at $1.65 \mathrm{e}-3 \mathrm{~s}$, a larger part of the surface switch in sliding (Fig. 12); the remaining part in sticking bear all the tangential stress, so that

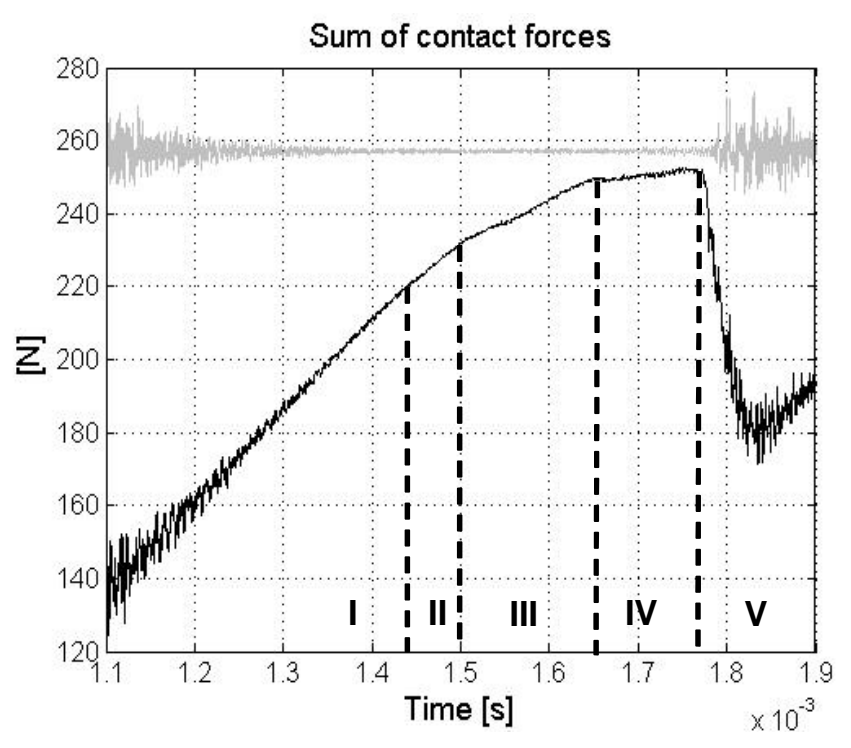

Figure 11. CONTACT FORCES BETWEEN 1.1e-3 s AND 1.9e$3 \mathrm{~s}$.

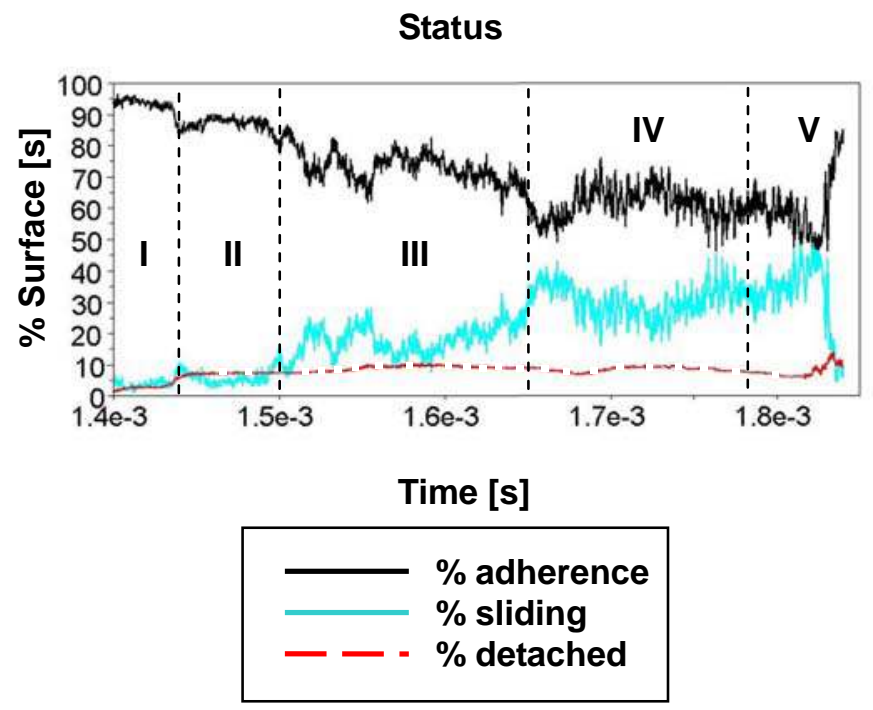

Figure 12. STATUS OF THE CONTACT POINTS ALONG THE ZONES I)-V).

the length of this zone is smaller and the sticking nodes reach the sliding condition in a shorter time.

Zone V): The remaining surface reaches the critical tangential stress and a larger percentage of the interface (more than 50\%) undergoes in sliding. The energy released activates rupture and waves propagation of greater magnitude, how can be seen in figure 13 where there are a series of head waves propagating towards the left (Fig. 13(a)) and a GR wave (generalized Rayleigh wave) propagating towards the right (Fig. 13(b)). The waves reach the boundaries of the system and are then reflected; thus, the waves generated at the interface 
excite the dynamics of the two bodies in sliding contact. After the macro-sliding phase the percentage of contact nodes in sticking status return to grow (Fig. 12 on the right).

The local status of the interface after every force drop, is directly affected by, the entity of the force drop: the more energy is released, the larger is the number of micro-slips zones.



\section{CONCLUSIONS}

The presented work deals with wave propagation and rupture nucleation when sliding initiates.

First, the dynamic rupture on a smooth interface between dissimilar materials has been investigated. The results obtained are consistent with the results found in literature [4] and the numerical model is validated. The nucleation causes the propagation of a slip pulse at generalized Rayleigh wave speed in the direction of sliding of the slowest velocity medium.

The roughness has been then introduced at the contact surfaces; the consequent heterogeneous stress distribution causes a more complex rupture process; Depending on the relative dimension between the roughness wavelength and the width of the wave fronts, two different behaviour can be observed: i) a coupling between the wave propagating into the two bodies, which causes the developing of two asymmetric rupture fronts in the opposite directions respect to the nucleation zone; ii) a decoupling of the wave propagation inside the two materials, characterized by an independent wave propagation and symmetry respect to the nucleation zone. In the second case the rupture profiles highlight different rupture fronts, propagating at different velocities inside the asperities. The velocities of the rupture fronts depend on the energy released at the nucleation. The presented results allow for asserting that the roughness at the interface plays a key role on wave propagation. A detailed parametrical analysis as a function of the roughness characteristics need to be carried out.

In a second time simulations have been performed to analyze the sliding initiation between the two contact surfaces.

It has been shown that the first phase of the sliding is characterized by several ramps of the global friction force followed by respective abrupt drops; while the ramps are characterized by micro-slip zones at the contact interface, the force drop is due to a macro-sliding of the contact surface. The elastic energy due to the global friction force is cumulated during the force ramp, until the next macro-sliding. A non continuous behaviour has been also observed inside a single force ramp, characterized by abrupt changes in the global force slope, and due to the different ratio of micro-slip zones at the contact surface. The results confirm the key role of the "precursor" [10], the micro-slips that occur at tangential global force well below the value expected from the friction law. Depending on the magnitude of the macro-sliding phase, waves propagate along the interface inside the corps in contact, affecting the status of the interface and exciting the dynamics of the system.

\section{REFERENCES}

[1] Adams G.G., (2001), "An intersonic slip pulse at a frictional interface between dissimilar materials", Journal of Applied mechanics, 68, pp. 81-86.

[2] Cochard A., Rice J.R., (2000), "Fault rupture between dissimilar materials: Ill-posedess, regularization and slippulse response", Journal of Geophysical Research, 105, pp. $25891-25907$.

[3] Shi Z., Ben-Zion Y., Needleman A., (2008), "Properties of dynamic rupture and energy partition in a solid with a frictional interface", Journ. Mech. Phys. Solids, 56, pp. 524.

[4] Andrews D.J., Ben-Zion Y., (1997), “Wrinkle-like slip pulse on a fault between different materials2, Journal of geophysical Research, 102 (10), pp. 553-571.

[5] Weertman J., (1980), "Unstable slippage across a fault separates elastic media of different elastic constant", Journal of Geophysical Research 85, pp.1455-1461.

[6] Ranjith K., Rice J.R., (2001), "Slip dynamics at an interface between dissimilar materials", Journal of the Mechanics and Physics of Solids 49, pp. 341-361. 
[7] Oden J.T., Martins J.A.C., (1985), "Models and computational methods for dynamic friction phenomena", Computer Methods in Applied Mechanics and Engineering 52, pp. 527-634.

[8] Adriaga R. M, (1983) "High frequency radiation from dynamic earthquake fault models", Annales Geophysicae 1, pp. 17-23.

[9] Das S., Aki K., (1977), "Fault plane with barriers: A versatile earthquake model", Journal of Geophysical Research 82, pp. 5658-5670.

[10] Rubinstein S.M. et al., (2007), "Dynamics of precursors to frictional sliding", Physical review letters 98, pp. 226103-1, 226103-4.

[11] Carpenter N.J., Taylor R.L., Kantona M.G., (1991), "Lagrange constraints for transient finite element surface contact", International Journal of Numerical Methods of Engineering, 32, pp. 103-128.

[12] Baillet L., Sassi T., (2005), "Mixed finite element formulation in large deformation frictional contact problem", Revue Européenne des Eléments Finis, 14 (23), pp. 287-304.

[13] Weertman J., (1963), "Dislocations moving uniformly on the interface between isotropic media of different elastic properties", Journal of Mech. Phys. Solids, 11, pp.197204.

[14] Ben-Zion Y., Azi K., (1990) "Seismic radiation from an SH line source in a laterally heterogeneous planar fault zone", Bull. Seismol. Soc. America, 80, pp. 971-994.

[15] Dunham E.M. et al., (2004), "Evidence for a supershear transient during the 2002 Denali fault earthquake", Bull. of Seism. Society of America, 94 No 6B, pp. S256-S268.

[16] Ampuero J.P., Ben-Zion Y., (2008), "Cracks, pulses and macroscopic asymmetry of dynamic rupture on a biomaterial interface with velocity-weakening friction", Geophys. J. Int., 173, pp. 674-692.

[17] Scheibert J. et al., (2008), "Micro-slip field at a rough contact driven towards macroscopic sliding", Arvix:0809.3188v1.

[18] Di Bartolomeo M., Meziane A., Massi F., Baillet L., Fregolent A., (2009), "Dynamic rupture at a frictional interface between dissimilar materials with asperities", Tribology International (submitted). 